\title{
Modification of the Basic Dilution Equation for the Programming of Serial Dilutions
}

Babu V. Bassa*

Rao M. Uppu

Author Affiliations:

Department of Environmental Toxicology, College of Sciences and Engineering, 108 Fisher Hall, James L. Hunt Street, Southern University and A\&M College, Baton Rouge, LA 70813.

* Corresponding author, Email address: bassa_babu@subr.edu

\begin{abstract}
Stock solutions made with accurately weighable quantities of biologically active compounds often do not result in physically measurable delivery volumes, delivered to cell cultures, assay buffers etc., consequently requiring delineation of the serial dilution steps of the stock solution in a case by case manner. The basic dilution equation $(\boldsymbol{C 1 V I}=\boldsymbol{C 2} V \mathbf{2})$ alone is not amenable for the programming and computerization of the serial dilutions. The best solution to this dilemma is to develop an equation with the delivery volume as one of the variables. We present here a modified dilution equation (MDA) that has the delivered volume as one of the variables. We demonstrate with examples how the equation can be used in delineating serial dilution steps either manually or through programming. The equation is $D=C 1 p / C 2 V 2$, where $D$ is the fold dilution required of a stock solution of $\boldsymbol{C} \boldsymbol{1}$ concentration which is diluted and delivered (pipetted) at a volume $\boldsymbol{p}$ to cell culture media, assay mixture etc., of volume $(\boldsymbol{V} \boldsymbol{2}-\boldsymbol{p})$, to achieve a final concentration of $\boldsymbol{C 2}$. The equation is useful when the volume of the stock solution made up to the final volume as calculated with basic dilution equation is not measurable physically.
\end{abstract}

Key words: Serial dilution; Basic dilution equation; Biosensors

\section{Introduction}

Many natural and synthetic principles like toxicants, antiserum, microbial field samples, forensic samples, hormones, and growth factors are present at ultra- low concentrations in the biological fluids. It is therefore necessary to handle those compounds at ultra-low concentrations during their chemical and biological assays. For example, the serum concentrations of hormones in humans are in the range of $10^{-12}-10^{-9} \mathrm{M}$. Furthermore, recently emerging biosensor assay methods have assay volumes as low as $5 \mu 1$, thus requiring smaller delivery volumes and greater degree of dilution. Stock solutions prepared with reliably weighable quantities of such compounds require customized dilution schemes to arrive at accurately measurable volumes pipetted, for example, into assay buffers or cell culture media. We present here a modified dilution equation that is readily amenable to computer programming of the serial dilutions.

\section{Methods}

\section{Modification of the basic dilution equation}

The newly proposed dilution equation is $\boldsymbol{D}=\boldsymbol{C 1} \mathbf{p} / \boldsymbol{C} 2 \boldsymbol{V} \mathbf{2}$, where $\boldsymbol{D}$ is the fold dilution required of a stock solution of $\boldsymbol{C} \boldsymbol{1}$ concentration which is diluted and delivered in a volume $\boldsymbol{p}$ to cell culture medium, assay mixture etc., of volume $(\boldsymbol{V} \boldsymbol{2}-\boldsymbol{p})$, to achieve a final concentration of $\boldsymbol{C} \boldsymbol{2}$. 
For the proof of the equation, first the total dilution factor $(C 1 / C 2)$ is written as a product of the final step of dilution where $\boldsymbol{p}$ volume is made up to $\boldsymbol{V} \boldsymbol{2}$ and the dilution required prior to that step $\boldsymbol{D}$ as shown below:

\section{Total dilution factor $=C 1 / C 2$}

2. Dilution at the final step where $p$ volume is made up to the final volume $V 2$ is $V 2 / p$. 3. Dilution required of the stock solution prior to the final step of dilution is $D$.

Therefore,

4. $C 1 / C 2=(D) *(V 2 / p)$

Then the equation is solved for $\boldsymbol{D}$.

5. $D=(C 1 / C 2) /(V 2 / p)$

6. $D=(C 1 / C 2) *(p / V 2)$

7. $D=C 1 p / C 2 V 2$

Therefore, the MDE is $\boldsymbol{D}=\boldsymbol{C 1} \boldsymbol{p} / \boldsymbol{C} \mathbf{2 V} \boldsymbol{2}$

where $\boldsymbol{D}=$ Times dilution required of the stock, $\boldsymbol{C} \boldsymbol{1}=$ Concentration of the stock solution, $\boldsymbol{C} \mathbf{2}=$ Final concentration required, $\boldsymbol{V} \mathbf{2}=$ Final volume (for example, assay or cell culture volume), and $\boldsymbol{p}=$ volume pipetted into the assay or cell culture dishes.

Note: In the general practice, the pipetted volume is very small compared to the final volume therefore, $\boldsymbol{P}$ is pipetted into the final volume in the test dishes and an equivalent volume of blank solvent (vehicle) is added to the control dishes.

Development and Implementation of a serial dilution algorithm using MDE

Once $\boldsymbol{D}$ is known, a serial dilution scheme can be sketched out by dividing $\boldsymbol{D}$ into a series of dilution steps. Since hundred fold dilutions $(10 \mu 1+990 \mu 1)$ are convenient and can be performed in an eppendorf tube, $\boldsymbol{D}$ can be converted into a few hundred-times dilutions and one Remaindertimes dilution. This procedure is illustrated with examples later in this communication. A typical code block is as follows:

While $P>=100$

$P=P / 100$

End While

Remainder $=P$

TextBox1.Text $=$ TextBox1.Text \& "Dilute the original stock" \& Remainder \& "times, that is" \& (1000) (1000/Remainder) \& "solvent +" \& (1000/Remainder) \& "stock."

While $P \geq 100$

$n=n+1$

$P=P / 100$

TextBox1.Text $=$ "Dilute stock" \& $n \&$ "100 times $(990 \mu l+10 \mu l)$ to make stock-" \& $(n+1)$

End While 
With the stock concentration $(\boldsymbol{C} 1)$, final required concentration $(\boldsymbol{C} 2)$, final assay or cell culture volume ( $\boldsymbol{V} \boldsymbol{2})$, and pipetted volume $(\boldsymbol{p})$ as inputs the same block of code produces instructions for a multitude of scenarios. An algorithm as outlined above was implemented in the Visual Basic programming language (1) to work on Windows-10 operating system and in App Inventor -2 language (2) to work on Android devices.

\section{Results and Discussion}

The Volume of the test solution pipetted into the cell cultures and assay buffers is often determined based on the pipetting devices available, the solubility of the test compound, and the chemical nature of the solvent in which the test compound is dissolved. Most test compounds in a cell biology laboratory are dissolved in dimethylsulfoxide. This requires severe restrictions of the volume of the delivered (pipetted) solution to prevent cyto-toxicity from the organic solvent.

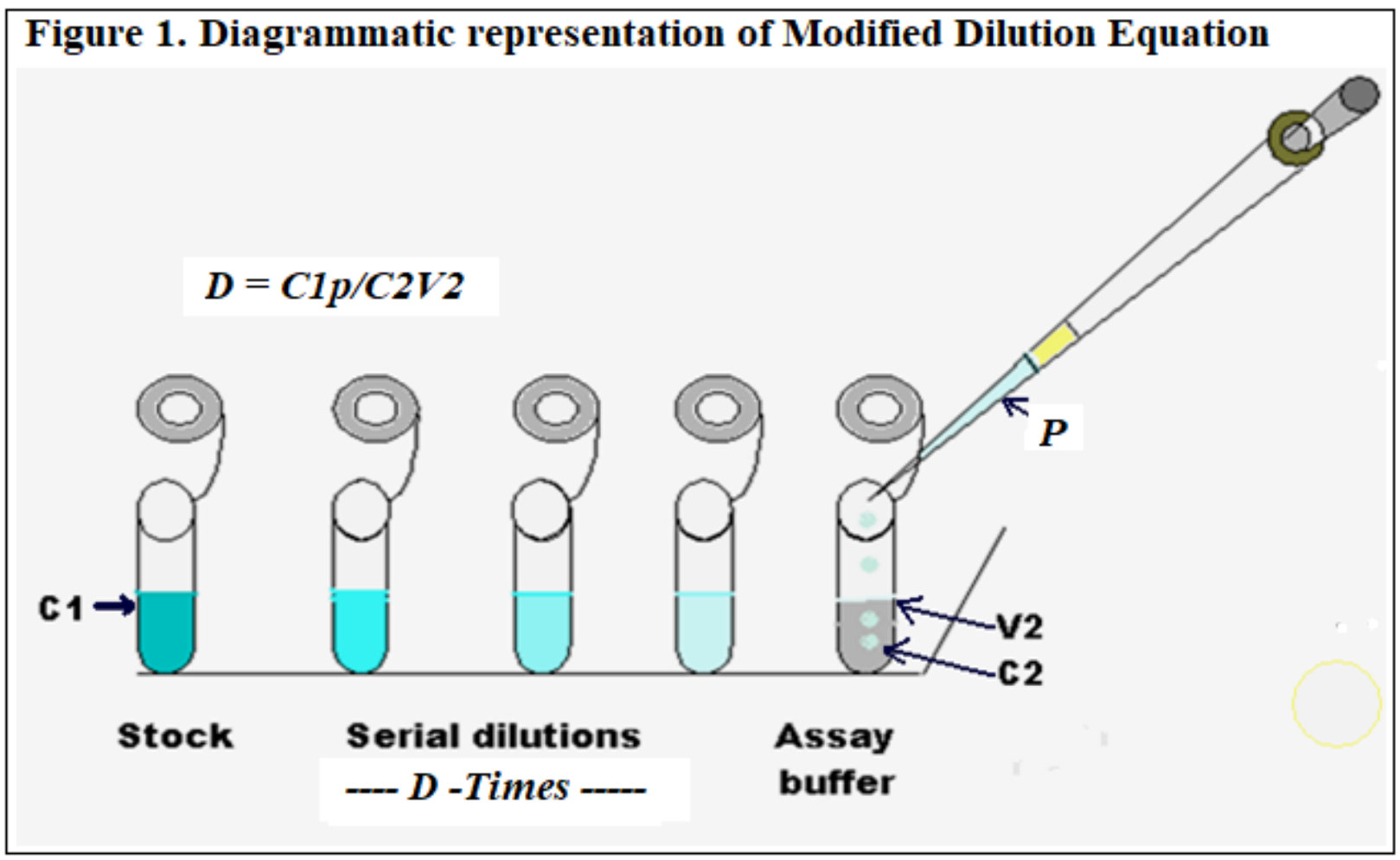

Figure 1. Legend. The principle of Modified Dilution Equation (MDE) is depicted in this diagram. The number of times that the stock needs to be diluted $(\boldsymbol{D})$ corresponding to a specified pipetted volume is calculated by the MDE. Based on the $\boldsymbol{D}$ the value the series of dilutions can be figured out either manually or through a computer program.

Upon deciding on the delivery volume, since single step dilutions are impractical and inaccurate, the dilutions are carried out serially. The current practice is to delineate the dilution steps by using the basic dilution equation, $\boldsymbol{C} \boldsymbol{V} \boldsymbol{V} \boldsymbol{I}=\boldsymbol{C} \mathbf{2} \boldsymbol{V}$, where $\boldsymbol{C} \boldsymbol{1}$ is the stock concentration, $\boldsymbol{V} \boldsymbol{1}$ is the volume of the stock solution that is made up to final volume $\boldsymbol{V} \mathbf{2}$ in order to achieve the final concentration $\boldsymbol{C 2}$, multiple times backwards from the pipetted volume. The dilutions are 
generally kept in the multiples of 10 scale (3). The current dilution practices lack uniformity between laboratories. In the present author's experience beginners in the biochemistry laboratory like students and clinicians practicing academic medicine have difficulty in delineating dilution schemes for various test compounds. Furthermore, programming of the serial dilution computations using the classic dilution equation is an impractical task because code has to be generated for each delivered and final volume combinations. A simple solution to this problem is to develop an equation that has the pipetted volume as one of the variables. We have presented here one such equation designated as modified dilution equation (MDE). The principle of this method is depicted in Figure 1. Two examples are presented (TextBox 1 and TextBox2) on the use of the program displaying the actual output from the windows/android apps. Each example also includes a verification of the dilution factor achieved following the serial dilution of the stock. The dilution factor for verification is calculated from the solution mixing instructions.

In conclusion the dilution equation described in this communication calculates the times-dilution of the stock solution required for a given pipetted volume and facilitates easy computation of the dilutions for agents active at ultra-low concentrations. Serial dilutions are regularly used in the biochemical, microbial, and serological laboratory work. Computerization of these calculations is necessary in order to prevent human errors. It is almost impossible to meaningfully program these calculations without an equation similar to the one proposed here. The computer programs developed using this equation can be used to calculate dilutions for reaction volumes as low as $5 \mu l$ as employed in biosensors (5) to volumes as high as several liters as employed in bioreactors (6). 
TextBox1. Example1. The concentration of free thyroxine in human blood is about $1.5 \mathrm{pg} / \mathrm{ml}$ (4). Given a stock solution of $5 \mathrm{mg} / \mathrm{ml}$ thyroxine, prepare standard solutions of $2.5 ; 5.0 ; 10 ;$ and $20 \mathrm{pg} / \mathrm{ml}$ concentrations in the final assay volume of $500 \mu \mathrm{l}$. Deliver the standards to the assay mixture at $5 \mu \mathrm{l}$ per tube.

Example1, performed manually using MDE:

$C 1=5,000,000,000 \mathrm{pg} / \mathrm{ml}$; Chosen $\mathrm{P}=5 \mu \mathrm{l}=0.005 \mathrm{ml} ; \mathrm{C} 2=20 \mathrm{pg} / \mathrm{ml} ; \mathrm{V} 2=500 \mu \mathrm{l}=0.5 \mathrm{ml}$.

$\mathrm{T}=(\mathrm{C} 1 \mathrm{~d}) /(\mathrm{C} 2 \mathrm{~V} 2)=(5000000000 * 0.0 .005) /(20 * 0.5)=2500000$ times

Repeat $\mathrm{T}=\mathrm{T} / 100$ until $\mathrm{T}<100$

$2500000 / 100=25000$

$25000 / 100=250$

$250 / 100=2.5$

Example1, Instructions written manually:

1. Dilute the original stock 2.5 times $(400 \mu \mathrm{l}+600 \mu \mathrm{l})$ to make Stock2.

2. Dilute stock2 100 times $(10 \mu \mathrm{l}+990 \mu \mathrm{l})$ to make Stock3.

3. Dilute stock3 100times $(10 \mu \mathrm{l}+990 \mu \mathrm{l})$ to make Stock4.

4. Dilute stock4 100times $(10 \mu l+990 \mu l)$ to make Stock5.

Further perform successive half dilutions of Stock5 to prepare Stock6, Stock7, and Stock8, for $10 ; 5$; and $2.5 \mathrm{pg} / \mathrm{ml}$ concentrations respectively and add $5 \mu \mathrm{l}$ per tube to respective tubes containing $245 \mu$ l assay buffer. Example1, Performed by the Windows/Android application using MDE:

Entries are $C 1=5 \mathrm{mg} / \mathrm{ml} ; d=5 \mu \mathrm{l} ; C_{2}=20,10,5,2.5 \mathrm{pg} / \mathrm{ml}$; and V2 $=500 \mu \mathrm{l}$.

(Note: All unit conversions and calculation of the $\mathrm{T}$ are done by the program)

\section{Windows/Android output:}

Dilute the original stock 2.50 times i.e. $400 \mu$ l stock $+600 \mu l$ solvent to make Stock1

Dilute stock1 100 times i.e. $(10 \mu \mathrm{l}+990 \mu \mathrm{l})$ to make stock2

Dilute stock2 100 times i.e. $(10 \mu \mathrm{l}+990 \mu \mathrm{l})$ to make stock3

Dilute stock3 100 times i.e. $(10 \mu l+990 \mu l)$ to make stock4

Designate Stock-4 as Final stock-F1

1. Mix Final stock-F1 $5 \mu$ l with $495 \mu$ l solvent/cell culture medium/reaction mixture etc., as appropriate for $20 \mathrm{pg} / \mathrm{ml}$ final concentration.

2. Dilute Stock-F1 $500 \mu l$ to $1000 \mu$ i.e. $500 \mu l$ Stock-F1 + 500 $\mu$ l solvent to make Stock-F2. Mix Stock-F2 $5 \mu \mathrm{l}$ with $495 \mu$ l solvent/reaction mixture for $10 \mathrm{pg} / \mathrm{ml}$ final concentration.

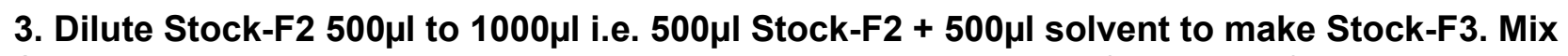
Stock-F3 $5 \mu \mathrm{l}$ with $495 \mu \mathrm{l}$ solvent/reaction mixture as appropriate for $5 \mathrm{pg} / \mathrm{ml}$ final concentration.

4. Dilute Stock-F3 $500 \mu l$ to $1000 \mu l$ i.e $500 \mu l$ Stock-F3 $+500 \mu l$ solvent to make Stock-F4. Mix Stock-F4 $5 \mu \mathrm{l}$ with $495 \mu \mathrm{l}$ solvent/reaction mixture for $2.5 \mathrm{pg} / \mathrm{ml}$ final concentration.

Verification:

$(\mathrm{C} 1 /$ net dilution $)=\mathrm{C} 2$

$5000000000 /(2.5 * 100 * 100 * 100) *(500 / 5)=5000000000 / 250000000=20 \mathrm{pg} / \mathrm{ml}$ (Highest concentration verified). 
TextBox.2. Example2. Titrate human insulin- immunized donkey serum for antihuman insulin antibody at 1000; 10,000; and 100;000 dilutions in a binding assay volume of $250 \mu$ l using radio-iodine labeled human insulin at 40,000 $\mathrm{cpm} / \mathrm{ml}$ final concentration. The stock insulin label contains $10 \mathrm{million} \mathrm{cpm} / \mathrm{ml}$ label. Keep the delivered volume at $20 \mu \mathrm{l}$ for the serum and $5 \mu \mathrm{l}$ for the insulin label.

Example2, Computations for the anti serum performed by the Windows/Applnventor2 program.

Entries: $C 1=1$ Anyting; $d=20 \mu \mathrm{l} ; C 2=1 / 10000 ; 1 / 100000 ; 1 / 1000000 ;$ V2 = 250 $\mathrm{l}$.

Windows/Android output for the anti-insulin-antibody titration:

Dilute the original stock 80.00 times i.e. $013 \mu l$ stock $+987.5 \mu l$ solvent to make Stock1.

Designate Stock-1as Stock-F1

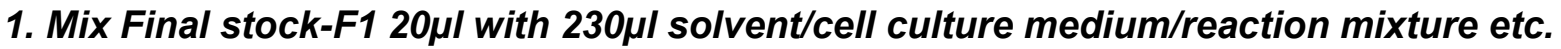
or spread on agar as appropriate for 1000Times final dilution.

2. Dilute Stock-F1 $100 \mu l$ to $1000 \mu l$ i.e. $100 \mu l$ Stock-F1 + $900 \mu l$ solvent to make Stock-F2

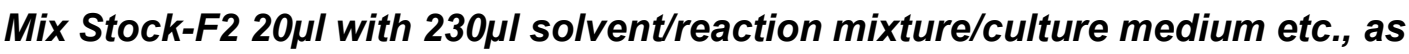
appropriate for 10000Times final dilution.

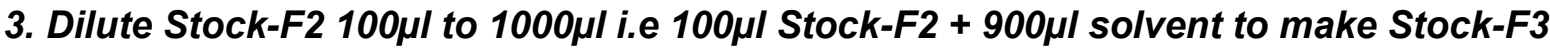

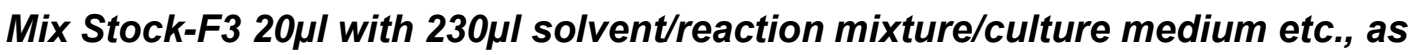
appropriate for 100000Times final dilution/reaction mixture/culture medium etc., as appropriate for 1000000Times final dilution.

Verification of Example -2 serum titration:

(C1/Total dilution) $=$ C2

$1 /((80) *(250 / 20))=1 / 1000$

(Highest of the final concentrations verified).

Example2 continued: Computations for the insulin label performed by the program: Entries are C1= $10000000 \mathrm{cpm} / \mathrm{ml} ; \mathrm{C2}=$ 40000cpm/ml; V2 = $250 \mu l ;$ and $d=5 \mu l$

Windows/Applnventor2 display:

Dilute the original stock 5.00 times i.e. $200 \mu$ l stock $+800 \mu l$ solvent to make Stock1. Designate Stock-1as Stock-F1

1. Mix Stock-F1 $5 \mu$ l with $245 \mu$ l solvent/cell culture medium/reaction mixture etc., as appropriate for $40000 \mathrm{cpm} / \mathrm{ml}$ final concentration.

Verification for Example-2 insulin label:

C1/Total dilution $=$ C2

$10,000,000 /((5) *(250 / 5))=10,000,000 / 250=40,000$

Note: In practice $5 \mu l$ label is added to control tubes as well as test tubes containing $250 \mu l$ assay buffer containing the anti serum. 


\section{References:}

1. https://visualstudio.microsoft.com > Visual Studio IDE | Microsoft Visual Studio

2. http://ai2.appinventor.mit.edu/

3. https://www.training.nih.gov/assets/Lab Math_II Transcript - 508.pdf

4. Kumar E, McCurdy MT, Koch CA, Hamadah A, Fülöp T, and Gharaibeh KA. (2018) Impairment of Thyroid Function in Critically Ill Patients in the Intensive Care Units. Am J Med Sci. 355(3):281-285

5. ai2.appinventor.mit.edu/

6. Singh P, Pandey SK, Singh J, Srivastava S, Sachan S, Singh SK (2016) Biomedical Perspective of Electrochemical Nanobiosensor. Nanomicro Lett.(3):193-203

7. Valdiani A, Hansen OK, Nielsen UB, Johannsen VK, Shariat M, Georgiev MI, Omidvar V, Ebrahimi M, Tavakoli Dinanai E, Abiri R (2018).

Bioreactor-based advances in plant tissue and cell culture: challenges and prospects. $\underline{\text { Crit }}$ Rev Biotechnol. 15:1-15

Conflicts of interest: None to declare.

Contributions:

Babu V. Bassa (Corresponding author): Derivation of the modified dilution equation (MDA), Preparation of the manuscript.

Rao M. Uppu: Preparation of the manuscript and compilation of the illustrations. 
